\title{
Long-term survival after chemoembolization of metastatic right atrial tumor thrombus as a presenting feature of hepatocellular carcinoma: A case study
}

\author{
JUN-HUI SUN, YUE-LIN ZHANG, CHUN-HUI NIE, LI-MING CHEN, \\ JIAN-DI HE, WEI-LIN WANG and SHU-SEN ZHENG
}

Division of Hepatobiliary and Pancreatic Surgery, Key Laboratory of Combined Multi-organ Transplantation, Ministry of Public Health Key Laboratory of Organ Transplantation of Zhejiang Province; Department of Surgery, First Affiliated Hospital, School of Medicine, Zhejiang University, Hangzhou 310003, P.R. China

Received December 15, 2011; Accepted February 17, 2012

DOI: $10.3892 / \mathrm{ol} .2012 .618$

\begin{abstract}
Advanced hepatocellular carcinoma (HCC) with invasion into the heart through the hepatic vein is a recognized rare occurrence with an extremely poor prognosis. Patients who present with right heart tumor thrombus have generally been considered inoperable. Although aggressive resection and liver transplantation treatment have previously been performed, the results remain unsatisfactory. However, HCC with extension into the heart usually indicates a contraindication for transcatheter arterial chemoembolization (TACE). In this study, a rare case of HCC with metastatic inferior vena cava (IVC) and right atrial (RA) tumor thrombus was reported. The young patient was admitted to our department due to Budd-Chiari syndrome. Following diagnosis according to CT image findings and laboratory data, the patient underwent TACE therapy. This treatment resulted in a marked reduction in the liver tumor and the right atrial tumor thrombus. Following TACE therapy, the patient survived for 3 years and 10 months and remains alive without any signs of recurrence. This case indicates that TACE therapy can be used successfully for the treatment of advanced $\mathrm{HCC}$ with heart tumor thrombus and may result in long-term survival.
\end{abstract}

\section{Introduction}

Hepatocellular carcinoma (HCC) is one of the most common types of malignant liver tumor, which is the third leading cause of cancer mortality worldwide (1-3). Studies have demonstrated that countries in Asia, particularly China, have

Correspondence to: Professor Shu-Sen Zheng, Division of Hepatobiliary and Pancreatic Surgery, Department of Surgery, First Affiliated Hospital, School of Medicine, Zhejiang University, 79 Qingchun Road, Hangzhou, Zhejiang 310003, P.R. China E-mail: shusenzheng@zju.edu.cn

Key words: chemoembolization, hepatocellular carcinoma, metastasis, tumor thrombus a high incidence rate of $\mathrm{HCC}$, due to endemic hepatitis $\mathrm{B}$ and $C(4,5)$. Surgeries, including complete surgical resection and liver transplantation, offer the best prognosis for $\operatorname{HCC}(6,7)$. However, $70-85 \%$ of patients with HCC are not surgical candidates due to advanced liver disease or extrahepatic metastases $(8,9)$. Metastatic right atrial (RA) tumor thrombus is rare in advanced HCC patients, but the prognosis is poor $(10,11)$. Transcatheter arterial chemoembolization (TACE) is the most widely used primary treatment for patients with HCC who are considered unsuitable candidates for surgery $(12,13)$.

We present a case of an HCC patient with metastatic RA tumor thrombus who presented with secondary Budd-Chiari syndrome. The patient underwent effective TACE therapy and remains alive after 3 years and 10 months.

\section{Patients and methods}

The patient was a 45-year-old man with a one-month history of edema in the lower extremities and recurrent episodes of chest distress. Examination revealed no abdominal pain, abdominal distension, or other manifestations of liver cancer.

Upon admission, a physical examination was conducted. It was discovered that both legs had marked edema, but no further abnormalities, including hepatosplenomegaly and ascites, were observed. Laboratory examinations revealed that the level of $\alpha$-fetoprotein was $2041.3 \mathrm{ng} / \mathrm{ml}$ (normal value, $<20.0 \mathrm{ng} / \mathrm{ml})$. The levels of glutamate oxaloacetic transaminase $44 \mathrm{U} / 1(<40 \mathrm{U} / \mathrm{l})$, glutamate pyruvate transaminase $34 \mathrm{U} / 1(<50 \mathrm{U} / \mathrm{l})$, total bilirubin $18.0 \mu \mathrm{mol} / 1(<22 \mu \mathrm{mol} / \mathrm{l})$, and albumin $31.4 \mathrm{~g} / 1$ (35-55 g/l) were elevated. The viral marker tests demonstrated that HBsAg was positive.

A chest X-ray revealed a moderate right pleural effusion, but no abnormalities were observed from the ECG. The abdominal-enhanced CT scan, which was performed on admission, demonstrated an arterial-phase enhancing mass in the left lobe which was found to have extended into the inferior vena cava (IVC) and RA (Fig. 1). The tumor and the RA tumor thrombus demonstrated an enhancing pattern of HCC. Therefore, a diagnosis of HCC with RA tumor thrombus was established based on the high level of plasma $\alpha$-fetoprotein 
(>400 ng/ml) combined with typical CT appearances. The CT images also revealed a moderate right pleural effusion.

Selective hepatic angiography (Fig. 2) showed hypervascular tumor staining of the left hepatic lobe, which was typical of $\mathrm{HCC}$, and extended into the IVC and RA through the hepatic vein. The feeding arteries of the tumor and tumor thrombus were derived from the left hepatic artery. A filling defect of the IVC and RA, and upward collateral veins, were revealed by inferior vena cavography. Therefore, the patient was diagnosed with HCC complicated by secondary Budd-Chiari syndrome, resulting from tumor thrombus of the venous system.

Following diagnosis, the patient underwent 2 sessions of TACE therapy over a period of 3 months. Depending on the arterial supply of the tumor identified by the arteriographies, a 2.7 French microcatheter (Terumo, Tokyo, Japan) was superselectively placed into the feeding arteries of the tumor and the tumor thrombus for selective embolization which was carried out with an embolization suspension consisting of pirarubicin (30 mg), oxaliplatin (200 mg), hydroxycamptothecine (20 mg) and iodized-oil (Lipiodol; Guerbet, Aulnay-sous-Bois, France).

The study was approved by the ethics committee of the First Affiliated Hospital, School of Medicine, Zhejiang University. Consent was obtained from the patient in this study.

\section{Results}

Following TACE therapy, the pleural effusion and edema of the lower extremities disappeared. The level of $\alpha$-fetoprotein gradually decreased to a normal level $(<20 \mathrm{ng} / \mathrm{ml})$. A CT scan was performed 2 years later (Fig. 3), which revealed that the tumor thrombus within the IVC and RA had shrunk and almost disappeared. The liver tumor also markedly decreased in size and almost disappeared on the CT images. Following therapy, the patient has been regularly followed up and demonstrates no signs of tumor progression and has continued to experience quality of life 3 years and 10 months following treatment.

\section{Discussion}

The incidence of $\mathrm{HCC}$ is on the increase worldwide due to the rise in hepatitis B and C infection $(2,4)$. Hepatic resection or liver transplantation is restricted to a small minority of patients with HCC $(8,9)$. Greatly-advanced HCC with involvement of the heart is rare $(14,15)$. The early diagnosis of HCC with tumor thrombus extending into the heart is occasionally difficult, particularly when patients do not demonstrate abdominal manifestations of liver cancer (16). In this case study, the patient had tumor thrombus within the IVC and RA, which caused secondary Budd-Chiari syndrome, and which was identified as a presenting feature of HCC. Despite progress in the treatment of liver cancer, greatly-advanced HCC with metastatic IVC and RA tumor thrombus has an extremely poor prognosis $(14,15)$. The median survival of patients with metastatic IVC and RA tumor thrombus, without effective therapy, is only 2-3 months (17). For HCC with metastatic IVC and RA tumor thrombus, no effective therapy has yet been reported. For these patients, surgical resection is often not complete or impossible. Previous studies on surgery for HCC with metastatic IVC and RA tumor thrombus have demonstrated disappointing results with a mean survival time of approximately 8 months (18).

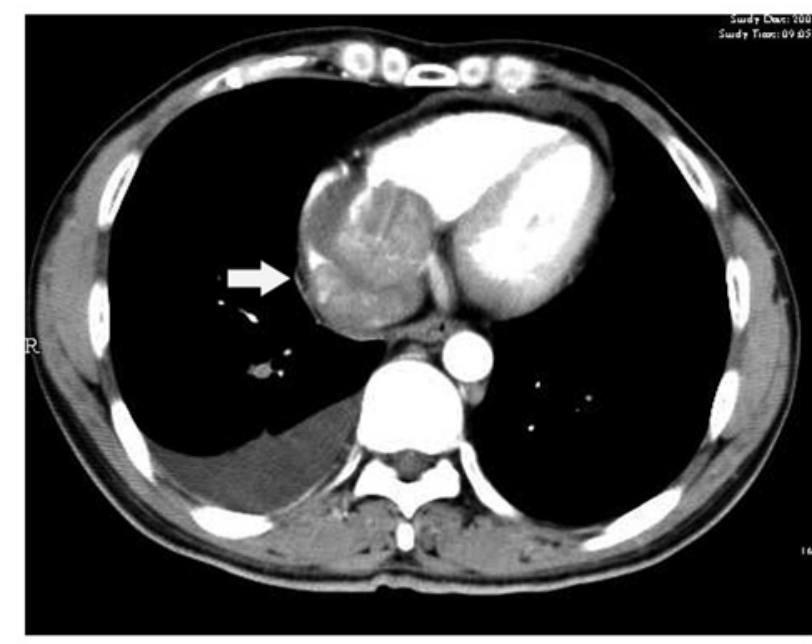

Figure 1. The abdominal-enhanced CT scan revealed an arterial-phase enhancing mass in the right atrial (arrow).

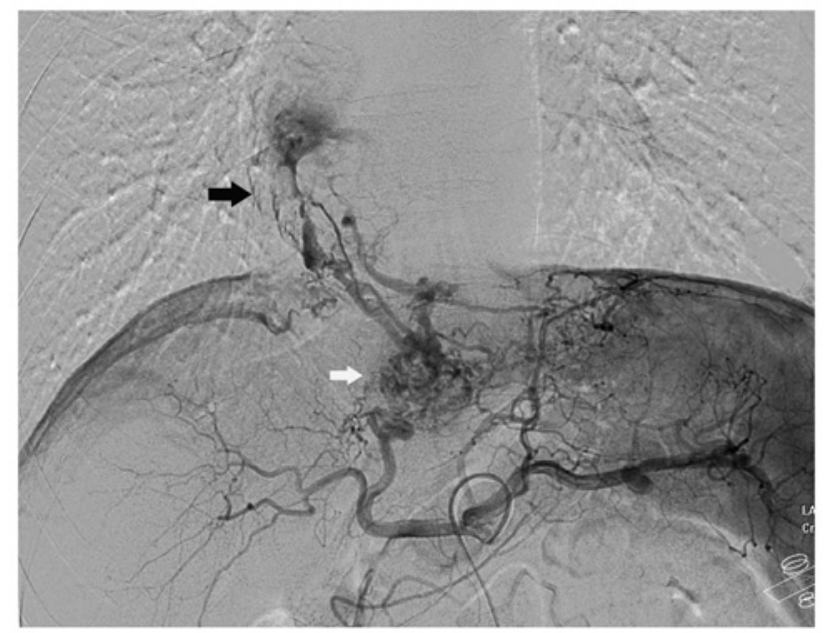

Figure 2. Hepatic angiography shows hypervascular HCC tumor staining of the left hepatic lobe (white arrow) which extended into the IVC and RA (black arrow) through the hepatic vein. HCC, hepatocellular carcinoma; IVC, inferior vena cava; RA, right atrial.

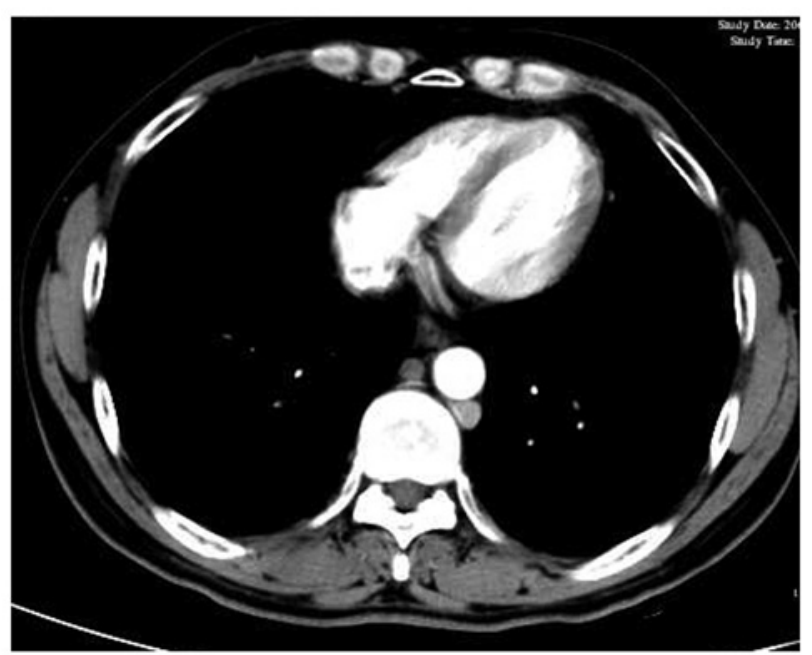

Figure 3. The abdominal-enhanced CT scan shows that the RA tumor thrombus shrank and almost disappeared. RA, right atrial. 
TACE has become the most widely used and effective treatment in patients with advanced $\mathrm{HCC}$, and provides a survival benefit for selective patients (19). It is also the most extensively used treatment for patients on the waiting list for liver transplantation (13). Following selective catheterization of the feeding artery of the tumor, a suspension consisting of iodized-oil and anticancer drugs was slowly injected into the target vessel to fulfill the microcirculation of the tumor, and remained there to prevent the blood flow causing tumor necrosis (13). TACE therapy has already become a significant and commonly used non-surgical treatment for HCC $(12,13,19)$.

However, there is a lack of large sample sizes in studies that have demonstrated the safety and efficacy of TACE in patients with greatly-advanced HCC with metastatic IVC and RA tumor thrombus. There have only been some case studies that have examined TACE therapy for HCC with metastatic IVC and RA tumors $(20,21)$. In this case study, celiac angiography and inferior vena cavography revealed that the feeding artery of the tumor thrombus associated with HCC was derived from the tumor vessels from the hepatic artery. Following selective chemoembolization, the decrease of the tumor thrombus and the liver tumor was clearly demonstrated by a CT scan. Additionally, the secondary Budd-Chiari syndrome of the patients due to the tumor thrombus disappeared.

In conclusion, our long-term survival case indicates that TACE therapy is not only an effective treatment against secondary Budd-Chiari syndrome due to tumor thrombus, but is also an effective treatment for advanced HCC with metastatic IVC and RA tumor thrombus, and is beneficial to long-term survival.

\section{Acknowledgements}

This study was supported by the National Natural Science Foundation of China (No. 30901446), the Medical Health Fund of Zhejiang Province (No. 2008A053), the Programme of Chinese Medical Science of Zhejiang Province (No. 2009CB040), the Program for Innovative Research Team of Science and Technology of Zhejiang Province (No. 2009R50038), the Major Program of the Science and Technology Bureau of Zhejiang Province (No. 2009C03012-1) and the National Major Program of Science and Technology of China (No. 2012ZX10002-17).

\section{References}

1. Parkin DM, Bray F, Ferlay J and Pisani P: Estimating the world cancer burden: Globocan 2000. Int J Cancer 94: 153-156, 2001.

2. He J, Gu D, Wu X, et al: Major causes of death among men and women in China. N Engl J Med 353: 1124-1134, 2005.
3. El-Serag HB: Hepatocellular carcinoma and hepatitis $\mathrm{C}$ in the Ued States. Hepatology 36: S74-S83, 2002.

4. Taura N, Hamasaki K, Nakao K, et al: Aging of patients with hepatitis $\mathrm{C}$ virus-associated hepatocellular carcinoma: Long-term trends in Japan. Oncol Rep 16: 837-843, 2006.

5. Llovet JM, Fuster J and Bruix J: Prognosis of hepatocellular carcinoma. Hepatogastroenterology 49: 7-11, 2002.

6. Kawano Y, Sasaki A, Kai S, et al: Short- and long-term outcomes after hepatic resection for hepatocellular carcinoma with concomitant esophageal varices in patients with cirrhosis. Ann Surg Oncol 15: 1670-1676, 2008.

7. Saab S, Yeganeh M, Nguyen K, et al: Recurrence of hepatocellular carcinoma and hepatitis B reinfection in hepatitis B surface antigen-positive patients after liver transplantation. Liver Transpl 15: 1525-1534, 2009.

8. Tsuzuki T, Sugioka A, Ueda M, Iida S, Kanai T, Yoshii H and Nakayasu K: Hepatic resection for hepatocellular carcinoma. Surgery 107: 511-520, 1990.

9. Fan HL, Chen TW, Hsieh CB, et al: Liver transplantation is an alternative treatment of hepatocellular carcinoma beyond the Milan criteria. Am J Surg 200: 252-257, 2010.

10. Florman S, Weaver M, Primeaux P, et al: Aggressive resection of hepatocellular carcinoma with right atrial involvement. Am Surg 75: 1104-1108, 2009

11. Lazaros G, Samara C, Nikolakopoulou Z and Tassopoulos N: Growth of hepatocellular carcinoma into the right atrium. A case of antemortem diagnosis with magnetic resonance imaging of the heart. Acta Cardiol 58: 563-565, 2003.

12. Scartozzi M, Baroni GS, Faloppi L, et al: Trans-arterial chemoembolization (TACE), with either lipiodol (traditional TACE) or drug-eluting microspheres (precision TACE, pTACE) in the treatment of hepatocellular carcinoma: efficacy and safety results from a large mono-institutional analysis. J Exp Clin Cancer Res 29: 164, 2010.

13. Baur M, Walter R, Gebauer A, et al: Chemoembolization with cisplatin, lipiodol and Gelfoam and subsequent systemic chemotherapy with cisplatin and interferon in patients with hepatocellular carcinoma: A non-randomized prospective study. Int J Oncol 23: 811-819, 2003.

14. Nam SW, Baek JT, Kang SB, et al: A case of the hepatocellular carcinoma during the pregnancy and metastasis to the left atrium. Korean J Hepatol 11: 381-385, 2005.

15. Chong VH, Jamaludin AZ, Lim KS, Abdullah HM and Nair RT: Hepatocellular carcinoma with intravascular and right atrial extension. Indian J Gastroenterol 27: 255, 2008.

16. Lazopoulos GL, Manns-Kantartzis M and Kantartzis MN: Severe right atrial obstruction due to cardiac extension of a hepatic carcinoma. Ann Thorac Surg 87: 645, 2009.

17. Zeng Z, Fan J, Tang Z, et al: A comparison of treatment combinations with and without radiotherapy for hepatocellular carcinoma with portal vein and/or inferior vena cava tumor thrombus. Int J Radiat Oncol Biol Phys 61: 432-443, 2005.

18. Asahara T, Itamoto T, Katayama K, et al: Hepatic resection with tumor thrombectomy for hepatocelluar carcinoma with tumor thrombi in the major vasculatures. Hepatogastroenterology 46: $1862-1869,1999$.

19. Takayasu K, Arii S, Ikai I, et al: Prospective cohort study of transarterial chemoembolization for unresectable hepatocellular carcinoma in 8510 patients. Gastroenterology 131: 461-469, 2006.

20. Dazai Y, Katoh T, Katoch I, Sueda S and Yoshida R: Effectiveness of chemoembolization therapy for metastatic right atrial tumor thrombus associated with hepatocellular carcinoma. Chest 96: 434-436, 1989.

21. Kashima Y, Miyazaki M, Kaiho T, et al: A successful treatment for hepatocellular carcinoma with atrial tumor thrombus. Hepatogastroenterology 43: 1040-1045, 1996. 